\title{
Magnitude of problematic anger and its predictors in the Millennium Cohort
}

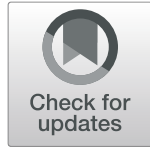

\author{
Amy B. Adler ${ }^{1}$, Cynthia A. LeardMann ${ }^{2,3^{*}}$, Kimberly A. Roenfeldt ${ }^{3}$, Isabel G. Jacobson ${ }^{2,3}$, David Forbes ${ }^{4}$, for the \\ Millennium Cohort Study Team
}

\begin{abstract}
Background: Problematic anger is intense anger associated with elevated generalized distress and that interferes with functioning. It also confers a heightened risk for the development of mental health problems. In military personnel and veterans, previous studies examining problematic anger have been constrained by sample size, cross-sectional data, and measurement limitations.
\end{abstract}

Methods: The current study used Millennium Cohort survey data $(N=90,266)$ from two time points $(2013$ and 2016 surveys) to assess the association of baseline demographics, military factors, mental health, positive perspective, and self-mastery, with subsequent problematic anger.

Results: Overall, $17.3 \%$ of respondents reported problematic anger. In the fully adjusted logistic regression model, greater risk of problematic anger was predicted by certain demographic characteristics as well as childhood trauma and financial problems. Service members who were in the Army or Marines, active duty (vs. reserves/national guard), and previously deployed with high levels of combat had increased risk for problematic anger. Veterans were also more likely to report problematic anger than currently serving personnel. Mental health predictors included posttraumatic stress disorder (PTSD), major depressive disorder (MDD), and comorbid PTSD/MDD. Higher levels of positive perspective and self-mastery were associated with decreased risk of problematic anger.

Conclusion: Not only did 1 in 6 respondents report problematic anger, but risk factors were significant even after adjusting for PTSD and MDD, suggesting that problematic anger is more than an expression of these mental health problems. Results identify potential targets of early intervention and clinical treatment for addressing problematic anger in the military and veteran context.

Keywords: Anger, Cohort studies, Military

\section{Background}

Anger, like any other emotion, is a normal human experience. In certain contexts, and when expressed appropriately, it may be useful. In an occupational context, anger may be an accepted emotion [1] and associated with benefits such as prosocial advocacy, promoting critical change, or improving subordinate performance [2].

\footnotetext{
* Correspondence: cynthia.a.leardmann.ctr@mail.mil

${ }^{2}$ Deployment Health Research Department, Naval Health Research Center,

140 Sylvester Road, San Diego, CA 92106, USA

3Leidos, 11951 Freedom Drive, Reston, VA, USA

Full list of author information is available at the end of the article
}

In the military, anger may be culturally acceptable, and many service members report that they believe anger is helpful to them in functioning, although high levels of anger have been associated with worse outcomes [3]. Depending on its frequency, intensity, and duration, anger can be considered "problematic anger" when it reaches a point where it is associated with elevated generalized distress, and begins to interfere with functioning [4]. Problematic anger may also impede interpersonal relationships particularly when expressed outwardly towards others [4]. For military personnel, problematic

C C The Author(s). 2020 Open Access This article is licensed under a Creative Commons Attribution 4.0 International License, which permits use, sharing, adaptation, distribution and reproduction in any medium or format, as long as you give appropriate credit to the original author(s) and the source, provide a link to the Creative Commons licence, and indicate if changes were made. The images or other third party material in this article are included in the article's Creative Commons licence, unless indicated otherwise in a credit line to the material. If material is not included in the article's Creative Commons licence and your intended use is not permitted by statutory regulation or exceeds the permitted use, you will need to obtain permission directly from the copyright holder. To view a copy of this licence, visit http://creativecommons.org/licenses/by/4.0/ The Creative Commons Public Domain Dedication waiver (http://creativecommons.org/publicdomain/zero/1.0/) applies to the data made available in this article, unless otherwise stated in a credit line to the data. 
anger may be a gateway to major disruption, both personally and professionally. In military and veteran personnel, problematic anger has been associated with mental health conditions, including posttraumatic stress disorder (PTSD [3, 5];), unhealthy habits and unnecessary risk taking [6], as well as relationship dysfunction [7]. Problematic anger and aggression are also risk factors for intent to harm others [5], interpersonal violence [8] and suicide-related outcomes [9]. While there is evidence that problematic anger is a significant concern in the civilian community [10], it is particularly important to understand the extent of problematic anger in military personnel and veterans and the key factors that contribute to its development and expression. The importance of this issue is heightened given the elevated risk of mental health difficulties in military personnel and veterans compared to civilians [11] and indications in meta-analyses that anger in veterans with PTSD is greater than that in civilians with PTSD [12].

Anger may be particularly relevant to the military in several ways. Not only does more problematic anger appear to be associated with combat exposure over time $[6,13]$, but anger frequently persists over the course of the post-deployment period [13], negatively impacting service members' ability to adjust following deployment and increasing their risk of developing PTSD [14, 15]. Besides this increased risk, problematic anger has important implications for clinical treatment, including that evidence-based treatment of PTSD may be less effective for veterans reporting high levels of co-occurring anger [16]. In addition, anger weakens PTSD treatment response in military veterans whether they have deployed to combat or peacekeeping missions [17]. In addition, anger has negative implications for job performance; anger-related reactions have been associated with unethical behavior during combat [18], alcohol problems [14], poor decision making [19], and blaming and retaliation [19]. Finally, it is important to consider the degree to which anger is a relatively acceptable emotion in the culture of the military [3], and thus may be reinforced in that occupational context.

Despite the evidence documenting the impact that problematic anger may have on individual health, relationships, and occupational functioning, the previous studies have been limited by small samples and crosssectional designs. Importantly, one cross-sectional study in the UK demonstrated the importance of demographic and mental health problems in estimating high anger scores in a large military sample [14]. The present study builds on these findings in several ways. First, the present study examined a large, diverse and comprehensive sample that includes veterans. Second, we introduced other risk factors such as history of sexual assault, combat severity, and financial problems along with potential mitigating variables. Third, by examining data collected at two points in time, we were able to assess the independent contribution of mental health problems reported at baseline in the modeling of problematic anger reported several years later. Finally, the present study also used a validated measure of anger that allows for an estimate of prevalence.

While the Spielberger State - Trait Anger Expression Inventory-2 (STAXI-2 [20]) is considered the gold standard for measuring anger, it is often too long for inclusion in studies designed to target a wide range of concerns. Therefore, the present study relied on the five-item $\mathrm{Di}$ mension of Anger Reactions scale (DAR-5 [21, 22]; that allowed for an estimate of prevalence of problematic anger. The DAR-5 has been validated against the STAXI-2 [20] and has an established cut-off to identify problematic anger in both military and civilian populations [21, 23].

The present study leveraged data from the Department of Defense's largest and longest ongoing prospective study of service members and veterans, the Millennium Cohort Study [24]. This first investigation of anger in the Millennium Cohort Study provides a unique opportunity to describe the magnitude of problematic anger in a large sample of service members and veterans, and to determine which individual and occupational factors are associated with problematic anger.

\section{Methods}

\section{Study population}

Established in 2001, the Millennium Cohort Study is a prospective cohort study investigating the long-term health effects related to military service both during service and after separation from the military [24]. The sample for the Cohort was randomly selected from US military rosters, with over-sampling of selected subgroups of interest, as previously described $[25,26]$. Service members were enrolled into four panels between 2001 and 2013, resulting in a total of 201,619 participants across all service branches, including both active duty and Reserves and National Guard (Reserve/Guard) members (27.3\% cumulative baseline response rate). Previous studies have found the Millennium Cohort to be a representative sample of service members in terms of health status, and analyses on weighting for nonresponse have not identified changes in metrics for mental disorders [27, 28]. Furthermore, using data from the Millennium Cohort, only slight differences between weighted means and nonweighted means for numerous mental and physical health conditions have been found; therefore, nonweighted data were used for the current study $[29,30]$.

After enrollment, participants were requested to complete follow-up surveys that are accessed online or via postal mail approximately every three to five years, 
even after they leave military service. These surveys collected information about behavioral, physical, and mental health, as well as service-related experiences. A detailed description of the Millennium Cohort has been previously published [24].

\section{Inclusion criteria}

Cohort members who completed both the 2011-2013 and 2014-2016 surveys, referred to as "baseline" and "follow-up" respectively for this study, were eligible $(n=$ 92,614) for the current study. Of these participants, those who were missing 3 or more of the items $(n=$ 2348) from the DAR-5 [21, 22] at follow-up were excluded from this study (analytic sample $N=90,266$, $97.5 \%$ of the eligible sample).

\section{Measures}

\section{Problematic anger}

Problematic anger was assessed at follow-up from the DAR-5, a validated instrument introduced on the follow-up survey (Cronbach's alpha $=0.91$ ). Participants responded to the question "Indicate the degree to which each statement describes your feelings or behavior:" with a 5-point Likert scale ranging from 1 (Not at all) to 5 (Very Much) for each of the five items (e.g., when I get angry, I get really mad; when I get angry at someone, I want to hit or clobber the person). Responses were summed and problematic anger (no/yes) was determined using the established cut-off of 12 points or higher [22]. The DAR-5 measure [21] has demonstrated robust convergent, concurrent and discriminant validity. In addition, the recommended cut off, aligned with the 75th percentile of the STAXI-2 [20], has been associated with psychological distress and functional impairment $[22,23]$ and utilized in numerous studies [31-33].

\section{Predictors of anger}

Demographics and military experiences Marital status and educational attainment were assessed at baseline using self-reported survey data. The other demographic and military characteristics were obtained at baseline from the Defense Manpower Data Center personnel files including age, sex, race/ethnicity, military service branch, military component, pay grade, and military separation status. Recent deployment experience in support of the operations in Iraq and Afghanistan was defined as occurring between baseline and follow-up. It was assessed using electronic deployment data from Defense Manpower Data Center combined with participants' responses to 13 self-reported combat experiences (e.g., "being attacked or ambushed", "receiving small arms fire") at follow-up. Those without a deployment between baseline and follow-up were classified as not recently deployed. Using methods similar to prior studies [34], individuals with a recent deployment were classified as deployed with no combat (endorsed 0 combat events), or deployed with low combat (endorsed 1-3 combat events), medium combat (endorsed 4-10 combat events), or high combat (endorsed 11-13 combat events). Due to the small number of participants reporting high levels of combat experiences, the two highest categories (medium and high) were combined into one category for this study.

Life stressors Childhood traumatic experiences (e.g. childhood sexual abuse) were asked for the first time of participants at the 2016 (follow-up) survey. These four items from the Juvenile Victim Questionnaire [35] assessed traumatic experiences that occurred prior to age 18, and were summed (0 to 4) [35]. Sexual assault was ascertained at baseline based on positive endorsement to one self-reported item (i.e., suffered forced sexual relations or sexual assault within the last 3 years). Financial problems were ascertained at baseline based on endorsement to one self-reported item (i.e., financial problems or worries within the last 4 weeks).

Psychological health/well-being Probable posttraumatic stress disorder (PTSD) was assessed at baseline using the PTSD Checklist-Civilian Version (PCL-C), used to rate the severity of 17 PTSD symptoms [36]. Based on criteria from the Diagnostic and Statistical Manual of Mental Disorders 4th edition (DSM-IV), participants were classified as having probable PTSD if they reported a moderate or greater level of at least one intrusion symptom, three avoidance symptoms, and two hyperarousal symptoms [37]. Probable major depressive disorder (MDD) was assessed at baseline using eight Patient Health Questionnaire (PHQ) items, which correspond to a depression diagnosis in the DSM-IV [37]. Based on criteria from the DSM-IV, probable MDD was defined as endorsing at least 5 items as "more than half the days" or "nearly every day", in which one symptom was depressed mood or anhedonia [38]. To assess the joint impact of PTSD and MDD, PTSD and MDD were combined to create a 4-level variable (neither PTSD nor MDD, PTSD only, MDD only, and comorbid PTSD and MDD). Problem drinking was assessed at baseline from endorsements of any of the 5 PHQ alcohol items (e.g., drank while working or taking care of responsibilities, missed or were late for work or other activities because you were drunk or hung over, drove a car after drinking too much) more than once in the last 12 months [39].

Potential mitigating factors for anger were also assessed. A version of the Posttraumatic Growth Inventory-Short Form (PTGI-SF) [40] was included at baseline to measure positive perspective. Unlike the 
original PTGI-SF, which asks for retrospective assessment of positive changes in personal perception following a traumatic event, the 11-item version used in the present study assessed current perspectives and did not reference a specific trauma. This current standing version was composed of the PTGI-SF [41] and an additional item about compassion for others. Mean scores were calculated based on participant responses to each item (e.g., "I know that I can handle difficulties," "I have a religious faith," "I have a sense of closeness with others") on a 6-point Likert scale from 0 (Not at all) to 5 (To a very great degree). Self-mastery was ascertained at baseline using 3 items (e.g., I can do just about anything I really set my mind to do) from Pearlin and Schooler's Self-Mastery Scale [42]. Mean scores were calculated based on responses on a 5-point Likert scale from 1 (Strongly disagree) to 5 (Strongly agree).

\section{Statistical analysis}

Descriptive analyses and chi-square tests were used to compare demographic, military, life stressors, and psychological health/well-being characteristics by problematic anger status. Bivariate logistic regression analyses were performed to assess the relationship between each factor (e.g., sex, age, marital status, educational attainment, race/ethnicity, military service branch, military service component, military separation status, recent combat severity, childhood traumatic experiences, sexual assault, financial problems or worries, mental health status, positive perspective, and self-mastery) and problematic anger. A multivariable logistic regression model was performed to determine which factors were significantly associated with problematic anger. In order to determine the influence of each factor above and beyond the other variables, all factors were included in the multivariable model regardless of statistical significance. To assess the percent of problematic anger that could be reduced in this population if certain exposures/factors were eliminated, the population attributable risk percent (PAR\%) was calculated for each life stressor and psychological/ well-being factor [the prevalence of the factor among those with problematic anger multiplied by the adjusted odds ratio (AOR) minus 1 divided by the OR multiplied by 100 (prevalence among cases $\times[(\mathrm{OR}-1) / \mathrm{OR}] \times$ $100 \%)]$. Multicollinearity was assessed using a variance inflation factor of four or higher. $P$-values of less than 0.05 were regarded as statistically significant. All analyses were completed using SAS statistical software, version 9.4 (SAS Institute, Inc., Cary, NC).

\section{Missing data}

Among the study participants $(n=90,266)$, each DAR-5 item had less than $0.4 \%$ missing. All model predictors had less than $3.1 \%$ missing with the exception of the childhood traumatic experiences. Each of the four items had approximately $3 \%$ missing and an additional $3 \%$ who responded "prefer not to answer." These responses were therefore classified into their own category to maintain these participants in analyses. For all other items, we used multiple imputation to maintain participants. Discriminant functions estimated categorical variables to ensure imputed data were integers within the range of possible values. A total of 50 imputed datasets were generated [43].

\section{Results}

Of the 90,266 study participants, the population was predominantly male (70.6\%), 26 to 39 years of age $(50.3 \%)$, non-Hispanic whites (76.9\%), and married (66.5\%). The largest proportion of participants had at least some college education (91.4\%), served in the Army (44.4\%), served on active duty (55.1\%), had separated from service by follow-up (62.3\%), and had not deployed between baseline and follow-up (88.4\%). Baseline characteristics are presented in Table 1. Overall, 15,600 (17.3\%) participants had problematic anger at follow-up. Notably, participants with problematic anger, compared to those without, were proportionally more likely to be younger ( $<40$ years old); less educated (high school or less); separated from the military, and in the Army or Marines (all $p<0.001)$. In addition, participants who experienced any type of life stressor (all $p<0.001$ ) or had a probable mental disorder $(\mathrm{p}<0.001)$ or problem drinking $(p<0.001)$ were proportionally more likely to report problematic anger (Table 1). Participants with problematic anger had lower mean scores for both positive perspective $\quad(\mathrm{p}<0.001)$ and self-mastery $\quad(\mathrm{p}<0.001)$ compared to those without problematic anger.

Appreciable endorsement of DAR- 5 items was calculated as any response from "moderately" to "a lot". Across participants, 24.3\% endorsed "I often find myself getting angry at people or situations", $22.0 \%$ endorsed "When I get angry, I get really mad", $14.8 \%$ endorsed "When I get angry, I stay angry", 10.8\% endorsed "When I get angry at someone, I want to hit or clobber the person", and 9.1\% endorsed "My anger prevents me from getting along with people as well as I'd like to". Responses to the DAR- 5 items by problematic anger status are presented in Fig. 1. For each item, endorsement was much higher among those with problematic anger.

Table 2 provides ORs for problematic anger at followup, after adjusting for all variables in the table. Given that there was no meaningful difference between results with and without multiple imputation, the imputed results are displayed. After adjustment, demographic factors found to be significantly associated with problematic anger included male sex, younger age, less educational attainment, black non-Hispanic and other race/ 
Table 1 Frequencies and Percentage of Baseline Characteristics by Problematic Anger Status

\begin{tabular}{|c|c|c|c|c|c|}
\hline \multirow[t]{2}{*}{ Baseline Characteristics* } & \multirow{2}{*}{$\begin{array}{l}\text { Study } \\
\text { Sample } \\
\text { n }\end{array}$} & \multicolumn{2}{|c|}{ No Problematic Anger } & \multicolumn{2}{|c|}{ Problematic Anger } \\
\hline & & $n$ & $\%$ & $n$ & $\%$ \\
\hline Sample & 90,226 & 74,626 & 82.7 & 15,600 & 17.3 \\
\hline \multicolumn{6}{|l|}{ Demographics } \\
\hline \multicolumn{6}{|l|}{ Sex } \\
\hline Male & 63,650 & 52,643 & 70.5 & 11,007 & 70.6 \\
\hline Female & 26,576 & 21,983 & 29.5 & 4593 & 29.4 \\
\hline \multicolumn{6}{|l|}{ Age } \\
\hline $17-25$ years & 9682 & 7438 & 10.0 & 2244 & 14.4 \\
\hline 26-39 years & 45,395 & 36,369 & 48.7 & 9026 & 57.9 \\
\hline 40 years or more & 35,149 & 30,819 & 41.3 & 4330 & 27.8 \\
\hline \multicolumn{6}{|l|}{ Educational attainment } \\
\hline High school or less & 7776 & 5673 & 7.6 & 2103 & 13.5 \\
\hline Some college/Associate's degree & 41,244 & 32,394 & 43.4 & 8850 & 56.7 \\
\hline Bachelor's degree & 23,132 & 20,030 & 26.8 & 3102 & 19.9 \\
\hline Graduate degree & 18,071 & 16,526 & 22.1 & 1545 & 9.9 \\
\hline \multicolumn{6}{|l|}{ Race/ethnicity } \\
\hline White, non-Hispanic & 69,361 & 57,973 & 77.7 & 11,388 & 73.0 \\
\hline Black, non-Hispanic & 8693 & 7059 & 9.5 & 1634 & 10.5 \\
\hline Other & 12,122 & 9549 & 12.8 & 2573 & 16.5 \\
\hline \multicolumn{6}{|l|}{ Marital status } \\
\hline Never married & 17,362 & 14,134 & 18.9 & 3228 & 20.7 \\
\hline Married & 59,961 & 50,415 & 67.6 & 9546 & 61.2 \\
\hline Formerly married & 12,903 & 10,077 & 13.5 & 2826 & 18.1 \\
\hline
\end{tabular}

\section{Military}

Service branch

Army
Navy/Coast Guard
Marine Corps
Air Force

Service component

Reserves/National Guard

Active duty

Military status at 2016

Not separated (currently serving)

Separated (veteran)

Recent deployment experience, 2013-2016 ${ }^{\mathrm{a}}$

Not recently deployed

Deployed, no combat

Deployed, low combat

Deployed, medium/high combat

\section{Life stressors}

Childhood traumatic experiences $^{b}$

None

1 experience
40,022

16,581

6622

27,001

40,540

49,686

33,974

56,252

79,718

3178

3130

1739

49,179

18,311
31,119

14,120

4834

24,553

33,897

40,729

29,755

44,871

65,203

2982

2764

1416
41.7

18.9

6.5

32.9

45.4

54.6

39.9

60.1

87.4

4.0

3.7

1.9
43,560

14,899
58.4

20.0
8903

57.1

15.8

11.5

15.7

2448

6643

42.6

57.4

27.0

73.0

11,381

14,515

93.0

196

1.3

2.3

2.1

323

5619

36.0

3412 
Table 1 Frequencies and Percentage of Baseline Characteristics by Problematic Anger Status (Continued)

\begin{tabular}{|c|c|c|c|c|c|}
\hline \multirow[t]{2}{*}{ Baseline Characteristics* } & \multirow{2}{*}{$\begin{array}{l}\text { Study } \\
\text { Sample } \\
\text { n }\end{array}$} & \multicolumn{2}{|c|}{ No Problematic Anger } & \multicolumn{2}{|c|}{ Problematic Anger } \\
\hline & & $n$ & $\%$ & $n$ & $\%$ \\
\hline 2 experiences & 11,336 & 8404 & 11.3 & 2932 & 18.8 \\
\hline 3 or more experiences & 2275 & 1394 & 1.9 & 881 & 5.6 \\
\hline Missing/prefer not to answer & 9125 & 6369 & 8.5 & 2756 & 17.7 \\
\hline \multicolumn{6}{|l|}{ Sexual Assault } \\
\hline No & 85,908 & 71,441 & 95.7 & 14,467 & 92.7 \\
\hline Yes & 2057 & 1388 & 1.9 & 669 & 4.3 \\
\hline \multicolumn{6}{|l|}{ Financial problems } \\
\hline Not bothered & 49,001 & 44,009 & 59.0 & 4992 & 32.0 \\
\hline Bothered a little & 29,351 & 23,441 & 31.4 & 5910 & 37.9 \\
\hline Bothered a lot & 10,942 & 6420 & 8.6 & 4522 & 29.0 \\
\hline \multicolumn{6}{|l|}{ Psychological health/well-being } \\
\hline \multicolumn{6}{|l|}{ Mental health status ${ }^{c}$} \\
\hline None & 79,553 & 69,699 & 93.4 & 9854 & 63.2 \\
\hline MDD only & 1152 & 671 & 0.9 & 481 & 3.1 \\
\hline PTSD only & 4928 & 2315 & 3.1 & 2613 & 16.8 \\
\hline Comorbid PTSD/MDD & 3588 & 1166 & 1.6 & 2422 & 15.5 \\
\hline \multicolumn{6}{|l|}{ Problem drinking ${ }^{d}$} \\
\hline No & 78,065 & 66,243 & 88.8 & 11,822 & 75.8 \\
\hline Yes & 9370 & 6129 & 8.2 & 3241 & 20.8 \\
\hline Positive Perspective (Mean \pm SD) $^{\mathrm{e}}$ & $3.7 \pm 0.9$ & $3.8 \pm 0.8$ & & $3.1 \pm 1.0$ & \\
\hline Self-Mastery $^{\mathrm{f}}($ Mean $\pm \mathrm{SD})$ & $4.0 \pm 0.7$ & $4.0 \pm 0.7$ & & $3.7 \pm 0.8$ & \\
\hline
\end{tabular}

${ }^{*}$ Characteristics are assessed at 2013 unless noted otherwise. Some columns ( $\mathrm{n}$ and \%) do not add up to column total due to missing data. Chi-square test of independence between each characteristic and problematic anger was statistically significant $(p<0.05)$, except sex

${ }^{\mathrm{a}}$ Based on deployments between baseline and follow-up in support of the operations in Iraq and Afghanistan

${ }^{\mathrm{b}}$ Based on 4 items assessed at follow-up: childhood sexual abuse, neglect, verbal abuse, and physical abuse

'Mental health status based on probable major depressive disorder (MDD) and/or posttraumatic stress disorder (PTSD) based on Diagnostic and Statistical Manual of Mental Disorders 4th edition (DSM-IV) criteria

${ }^{\mathrm{d}}$ Based on endorsing any of the five Patient Health Questionnaire (PHQ) alcohol items

${ }^{\mathrm{e}}$ Assessed from the mean score of all 11 items from the Current State -Posttraumatic Growth Index - Short Form (C-PTGI-SF)

${ }^{f}$ Assessed from the mean score of 3 self-mastery items from Pearlin and Schooler's Self - Mastery Scale

ethnicities (compared with white non-Hispanic), and marital status. During the model building process a significant interaction between marital status and age was discovered $(p<.01)$, which indicated that married and formerly married participants were significantly more likely to have problematic anger in the younger age groups (17-39 years old), but that marital status was not associated with problematic anger among the oldest participants (40+ years old). For military factors, Navy/ Coast Guard $[\mathrm{AOR}=0.65 ; 95 \% \mathrm{CI}: 0.61,0.69]$ and Air Force $[\mathrm{AOR}=0.52,95 \% \mathrm{CI}: 0.49,0.55]$ members were significantly less likely than Army personnel to have problematic anger. Active duty service members were significantly more likely to have problematic anger $[\mathrm{AOR}=1.28$; 95\% CI 1.23, 1.34] compared with Reservists and National Guardsmen. Separation from military service was also associated with higher odds of problematic anger $[\mathrm{AOR}=1.45 ; 95 \% \mathrm{CI} 1.38,1.52]$, as was deployment with medium/high combat severity between baseline and follow-up [AOR $=1.34 ; 95 \%$ CI 1.16, 1.54]. In a sub-analysis, when combat was categorized as a 3level variable (not recently deployed, deployed without combat, and deployed with combat), those who experienced any combat had increased odds for problematic anger $[\mathrm{AOR}=1.13,95 \% \mathrm{CI} 1.02,1.24]$.

All life stressors were significantly associated with problematic anger except for sexual assault. As the number of reported childhood traumatic experiences increased, so did the magnitude of the association with problematic anger, such that the strongest association was observed for those reporting 3 or more experiences $[\mathrm{AOR}=2.70 ; 95 \%$ CI 2.43, 3.00; PAR\%, 3.6] compared with those reporting no childhood trauma. A similar pattern was observed among those who reported financial problems or worries, with those reporting being "bothered a lot" having the strongest association [AOR = 


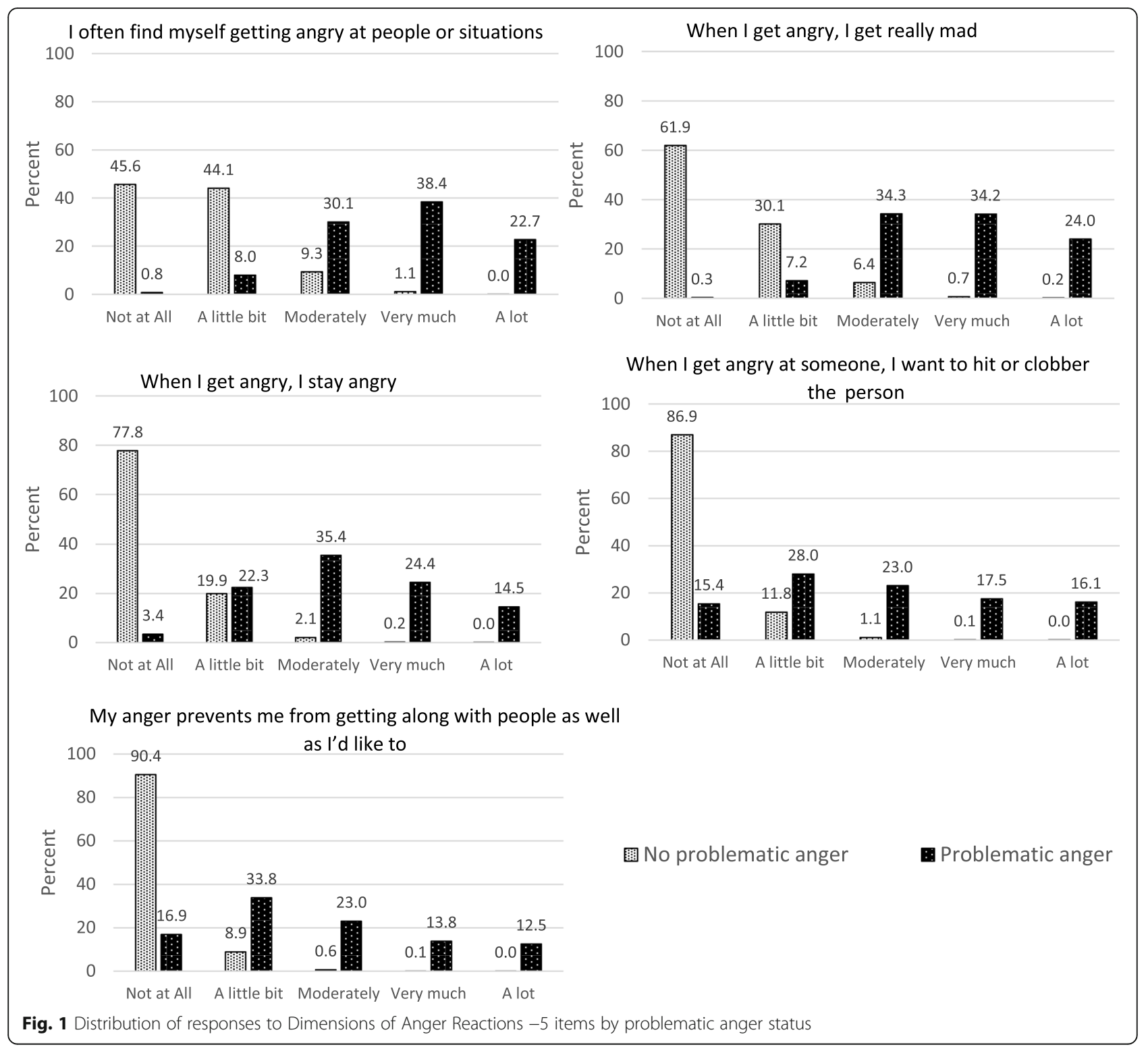

1.94, 95\% CI 1.82, 2.06; PAR\%, 14.0] with problematic anger compared with those who were not bothered. Significant associations were observed for all psychological health/well-being factors. The strongest magnitude of association was among those with comorbid PTSD and $\mathrm{MDD}[\mathrm{AOR}=4.43 ; 95 \% \mathrm{CI} 4.09,4.80 ; \mathrm{PAR} \%, 12.0]$ and those with PTSD only $[\mathrm{AOR}=3.52$; $95 \%$ CI 3.28, 3.78; $\mathrm{PAR} \%, 12.0]$. Problem drinking was also associated with problematic anger $[\mathrm{AOR}=1.46 ; 95 \%$ CI 1.38, 1.55; PAR\%, 6.6]. With regard to mitigating factors, as mean scores for positive perspective and self-mastery increased, odds for problematic anger significantly decreased. Specifically, for each one-unit increase in mean score for positive perspective, there was an $28 \%$ decrease in odds for problematic anger (PAR\%, 12.1 for a mean score increase from "moderate degree" to "a very great degree"). For every one-unit increase in mean score for self-mastery, there was a $16 \%$ decrease in odds for problematic anger (PAR\%, 9.3 for a mean score increase from "neither agree or disagree" to "strongly agree").

\section{Discussion}

The present study documents that more than one in six responders to the 2016 Millennium Cohort Study survey reported levels of anger that are considered to be problematic based on the DAR- 5 cutoff score of 12 points or higher. These findings provide an unprecedented examination of the scope of problematic anger in a robust sample of service members and veterans.

The present study also supports previous work that has found factors such as male gender, younger age, lower educational attainment, non-white race/ethnicity, 
Table 2 Adjusted ${ }^{a}$ Odds Ratios of Problematic Anger

Characteristic
Demographics
Male
Age (ref: $40+$ years old)
17-25 years old
26-39years old
Educational attainment (ref: graduate degree)
High school or less
Some college or Associate's degree
Bachelor's degree
Race/ethnicity (ref: white, non-Hispanic)
Black, non-Hispanic
Other
Marital status ${ }^{\mathrm{b}}$ (ref: never married)
Married
Formerly married
AOR $(95 \% \mathrm{Cl})$

$1.07(1.02,1.12)$

$1.79(1.66,1.93)$

$1.69(1.61,1.77)$

$1.98(1.81,2.15)$

$1.67(1.56,1.78)$

$1.26(1.17,1.35)$

$1.22(1.14,1.31)$

$1.20(1.14,1.27)$

$1.21(1.14,1.27)$

$1.10(1.03,1.18)$

Military service

Service branch (ref: Army)

$\begin{array}{ll}\text { Air Force } & 0.52(0.49,0.55) \\ \text { Navy/Coast Guard } & 0.65(0.61,0.69) \\ \text { Marine Corps } & 1.02(0.95,1.09)\end{array}$

Service component (ref: Reserves/National Guard)

Active duty

$1.28(1.23,1.34)$

Military status at 2016 (ref: not separated/currently serving)

Separated (veteran)

$1.45(1.38,1.52)$

Recent deployment experience, 2013-2016 (ref: not recently deployed)

Deployed, no combat

$0.57(0.48,0.67)$

Deployed with low combat

$1.00(0.88,1.13)$

Deployed with medium/high combat

$1.34(1.16,1.54)$

\section{Life stressors}

Childhood traumatic experiences $^{\mathrm{d}}$ (ref: none)

1 experience
2 experiences
3 or more experiences
Missing/prefer not to answer
Sexual assault (ref: no)
Yes

$1.45(1.38,1.53)$

$1.97(1.86,2.08)$

$2.70(2.43,3.00)$

$2.11(1.99,2.25)$

$1.09(0.97,1.23)$

Financial problems (ref: not bothered)

Bothered a little

$1.43(1.37,1.50)$

Bothered a lot

$1.94(1.82,2.06)$

\section{Psychological health/well-being}

Mental health status ${ }^{\mathrm{e}}$ (ref: none)

MDD only

$2.12(1.86,2.42)$

PTSD only

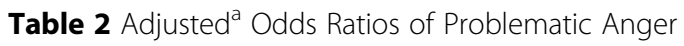

(Continued)

\begin{tabular}{ll}
\hline Characteristic & AOR $(95 \% \mathrm{Cl})$ \\
\hline Comorbid PTSD/MDD & $4.43(4.09,4.80)$ \\
Problem drinking (ref: no $)^{f}$ & \\
$\quad$ Yes & $1.46(1.38,1.55)$ \\
Positive Perspective $^{g}$ & $0.72(0.70,0.74)$ \\
Self-Mastery $^{\text {h }}$ & $0.84(0.82,0.87)$ \\
\hline
\end{tabular}

${ }^{a}$ Data are reported from one model that adjusted for all variables in this table

${ }^{\mathrm{b}} \mathrm{A}$ significant interaction with age and marital status was found, which

indicated that marital status was not significantly associated with problematic anger among the $40+$ year olds

' Based on deployments between baseline and follow-up in support of the operations in Iraq and Afghanistan

'Based on 4 items assessed at follow-up: childhood sexual abuse, neglect, verbal abuse, and physical abuse

${ }^{\mathrm{e}}$ Mental health status based on probable major depressive disorder (MDD) and/or posttraumatic stress disorder (PTSD) based on Diagnostic and Statistical Manual of Mental Disorders 4th edition (DSM-IV) criteria

${ }^{f}$ Based on endorsing any of the five Patient Health Questionnaire (PHQ) alcohol items

${ }^{9}$ Assessed from the mean score of all 11 items from the Current StatePosttraumatic Growth Index - Short Form (C-PTGI-SF); ORs refer to a 1 point change in mean score

${ }^{\mathrm{h}}$ Assessed from the mean score of 3 self-mastery items from Pearlin and Schooler's Self - Mastery Scale; ORs refer to a 1 point change in mean score

and childhood trauma to be associated with anger [14, $44,45]$. Notably, in the case of non-white race/ethnicity, anger may be a reflection of longstanding social inequality or other forms of discrimination without the societally-sanctioned opportunity to express outrage [46]. While not the focus of the present study, these findings highlight the importance of considering differences in the way anger is understood within a diverse military community.

In addition to corroborating findings from other research, this study identified that being married, financial problems, and military-specific factors were significantly associated with higher odds for problematic anger, including serving on active duty, deployment with high levels of combat experience, and separation from military service. Importantly, these relationships remained significant after adjusting for baseline mental health disorders, including PTSD and MDD, suggesting that problematic anger appears to be more than simply an expression of PTSD and MDD. Instead, problematic anger appears to manifest in individuals with a constellation of factors reflecting personal background, aspects of the military experience, financial concerns, and mental health problems. Some of these factors may be temporary (e.g., financial concerns, mental health concerns), although the exact duration of predictors is beyond the scope of the present study.

Perhaps one benefit of identifying the significant and independent relationships between a range of predictors and problematic anger is that there is a multitude of potential points for targeting interventions. The PAR\%s 
provide insight regarding which factors may be most influential in reducing problematic anger in this population. For example, by preventing or eliminating some of these modifiable or treatable conditions, such as financial problems, problem drinking, and PTSD, problematic anger may be reduced by $14.0,6.6$, and $12.0 \%$ respectively, assuming that these observed associations are causal and that elimination of these risk factors do not affect the distribution of other covariates. Conversely, increasing positive perspective and self-mastery (i.e., from mean score of 3 ["moderate agree" or "neither agree or disagree"] to 5 ["a very great degree" or "strongly agree"]) could lead to a reduction in problematic anger by 12.1 and $9.3 \%$, respectively. While other ORs had a large magnitude, such as childhood trauma and MDD, the PAR\% demonstrates that reductions in these factors would have a smaller effect $(<3 \%$ reduction) due to their lower prevalence in this population. These findings indicate that the effective prevention and treatment of modifiable conditions, such as problem drinking and PTSD, as well as the enhancement of positive perspective and self-mastery may reduce the prevalence of problematic anger.

Such interventions should be developed for militaryspecific application given the unique occupational context in which service members operate. Notably, the military occupational context trains individuals to maintain vigilance to threat and to consider confrontational strategies in the face of threat [47], which can potentially exacerbate anger. Not only should interventions take this context into account, but adaptation of existing interventions for the military context can be particularly beneficial since service members are more likely to engage in an intervention they regard as tailored for their needs [48].

Thus, developing interventions that target problematic anger in the military is critical given its high prevalence, distinction from other mental disorders, role in impeding effective PTSD treatment, and impact on vocational and interpersonal functioning. Results also suggest that directly targeting problematic anger may reduce the risk for interpersonal aggression and family violence, given research indicating that elevations on the DAR-5 conferred a 13 fold risk of aggression [Cowlishaw, Metcalf, Varker, Stone, Alkemade, Molyneaux, Gibbs, Block, Harms, MacDougall, Gallagher, Bryant, LawrenceWood, Kellett, O'Donnell and Forbes: Problematic anger prevalence and risk for aggression and suicidality in a post-disaster context, submitted]. The current findings further reinforced this elevated risk with over one in 10 respondents endorsing that when they get angry at someone, they wanted "to hit or clobber the person." Direct targeting of anger also potentially opens the gateway to improved outcomes in co-occurring conditions such as military-related PTSD. It will be important for future research to distinguish between predictors of long-held problematic anger and recently established problematic anger in order to develop optimally tailored intervention strategies. Although there has been little empirical attention paid to the development and testing of direct interventions for problematic anger in military populations, approaches to the management and treatment of problematic anger in military populations have been recently developed and pilot tested in both the US [49] and Australia [47]. Implementing these kinds of specific interventions may also help mitigate the poorer treatment outcomes found with military veterans with PTSD relative to their civilian counterparts [50].

Besides clinical treatment for pathological responses, early intervention programs can also be developed for lower intensity expressions of anger. Early intervention strategies could be inserted into nontraditional contexts such as preparing financial counselors to reinforce military-specific anger management techniques with service members bothered by financial problems [48]. Early interventions could also include training counselors who focus on the pivotal process of transitioning from military to veteran status in targeted anger management. Such initiatives like the US Army's Transition Assistance Program could integrate these techniques into their formal courses. Early interventions could also be adapted for military leaders, given the importance of leaders in establishing unit culture and influencing soldier health and wellbeing [51]. Results also highlight potential candidates for screening efforts and mental health treatments that are adapted to target anger as a primary goal.

The findings of the present study also point to another area of potential intervention, currently untapped in the conceptualization of anger responses and intervention. Specifically, findings indicate that individual endorsement of positive perspective and self-mastery is associated with reduced odds of problematic anger years later. These attitudes reflect the ability to use coping skills [52] that place challenges into perspective and that reflect the importance of finding meaning in one's life and work. Skills related to these concepts could be strengthened in service members directly through training [52, 53], and indirectly through institutional messaging about the importance of positive perspective and self-mastery, and through leaders who set an example and reinforce these attitudes. By supporting these kinds of adaptive skills and perceptions, studies could examine whether the risk of problematic anger years later may be lowered.

While the present study examines factors associated with problematic anger over a 3-4 year follow-up period using a large sample, there are limitations. These data are correlational and there was no measure of DAR- 5 on the baseline (2013) survey, which prevented the analysis of modeling the emergence of problematic anger or distinguishing between long-held and recently established 
problematic anger. In addition, self-reported survey data may be subject to recall and reporting biases. Finally, while the Millennium Cohort Study includes participants from all branches of service, active and Reserve components, and veterans, it may not necessarily be representative of the entire U.S. military or those who deploy. However, investigations of the Millennium Cohort Study have not demonstrated systematic sampling bias $[28,54]$.

\section{Conclusion}

Results from this prospective study underscore the relatively high prevalence of problematic anger in a large military sample and the markers of risk and resilience that can potentially influence problematic anger over a period of time. By reducing the risk of problematic anger, individuals and organizations like the military may be able to benefit in terms of improved employee health, relationships with family, friends and coworkers, occupational functioning and reduced risks for aggression and violence.

\section{Abbreviations \\ PTSD: Posttraumatic stress disorder; STAXI-2: State - Trait Anger Expression Inventory - 2; DAR-5: Dimension of Anger Reactions scale; PCL-C: PTSD Checklist-Civilian version; DSM-IV: Diagnostic and Statistical Manual of Mental Disorders 4th edition; MDD: Major depressive disorder; PTGI-SF: Posttraumatic Growth Inventory-Short Form; PAR\%: Population attributable risk percent: AOR: Adjusted odds ratio; Cl: Confidence interval}

\section{Acknowledgements}

In addition to the authors, Millennium Cohort Study team includes Richard Armenta, PhD; Satbir Boparai, MBA; Felicia Carey, PhD; Toni Rose GeronimoHara, MPH; Claire Kolaja, MPH; Rayna Matsuno, PhD; Deanne Millard; Chiping Nieh, PhD; Ben Porter, PhD; Teresa Powell, MS; Anna Rivera, MPH; Rudolph Rull, PhD; Beverly Sheppard; Daniel Trone, PhD; Jennifer Walstrom; and Steven Warner, MPH. The authors also appreciate contributions from the Deployment Health Research Department, Millennium Cohort Family Study, the Birth and Infant Health Research Team, and the Henry M. Jackson Foundation. We thank the Millennium Cohort Study participants. Disclaimer: I am a military service member or employee of the U.S. Government. This work was prepared as part of my official duties. Title 17, U.S.C. \$105 provides that copyright protection under this title is not available for any work of the U.S. Government. Title 17, U.S.C. \$101 defines a U.S. Government work as work prepared by a military service member or employee of the U.S. Government as part of that person's official duties.

\begin{abstract}
Authors' contributions
AA drafted the introduction and discussion; $C L$ drafted sections of the manuscript, conducted statistical analysis, and supervised the analysis; KR conducted the statistical analysis and drafted the methods and results. All authors helped with designing the study, interpreted the results, provided critical analysis of the project, critically reviewed the manuscript, and approved of the final version.
\end{abstract}

\section{Funding}

The Millennium Cohort Study and this project, Report Number 19-77, was supported and funded by the Military Operational Medicine Research Program, Defense Health Program, and Department of Veterans Affairs under work unit no. 60002. The views expressed in this article are those of the authors and do not necessarily reflect the official policy or position of the Department of the Navy, Department of Defense, nor the U.S. Government. The funders had no role in study design, data collection and analysis, decision to publish, or preparation of the manuscript.

\section{Availability of data and materials}

The data that support the findings of this study are not currently publicly available due institutional regulations protecting service member survey responses but are available from the corresponding author on reasonable request (may require data use agreements to be developed).

\section{Ethics approval and consent to participate}

The study protocol was approved by the Naval Health Research Center Institutional Review Board in compliance with all applicable Federal regulations governing the protection of human subjects. Research data were derived from an approved Naval Health Research Center, Institutional Review Board protocol number NHRC.2000.0007. All participants provided voluntary, informed written consent.

\section{Consent for publication}

Not applicable.

\section{Competing interests}

The authors declare no potential conflicts of interest with respect to the research, authorship, and/or publication of this article.

\section{Author details}

${ }^{1}$ Walter Reed Army Institute of Research, Silver Spring, MD, USA. ${ }^{2}$ Deployment Health Research Department, Naval Health Research Center, 140 Sylvester Road, San Diego, CA 92106, USA. 'eidos, 11951 Freedom Drive, Reston, VA, USA. ${ }^{P}$ Phoenix Australia - Centre for Posttraumatic Mental Health, Department of Psychiatry, University of Melbourne, Melbourne, Australia.

Received: 22 November 2019 Accepted: 5 July 2020

Published online: 27 July 2020

\section{References}

1. Gibson DE. Emotion scripts in organizations: a multi-level model. In: Ashkanasy N, Cooper C, editors. Research companion to emotion in organizations. London: Edward Elgar Publishers; 2008. p. 263-83.

2. Geddes D, Callister RR, Gibson DE. A message in the madness: functions of workplace anger in organizational life. Acad Manag Perspect. 2020;34(1):2847.

3. Adler AB, Brossart DF, Toblin RL. Can anger be helpful?: soldier perceptions of the utility of anger. J Nerv Ment Dis. 2017;205(9):692-8.

4. Novaco RW. The Novaco anger scale and provocation inventory (NAS-PI). Los Angeles: Western Psychological Services; 2003.

5. Gonzalez Ol, Novaco RW, Reger MA, Gahm GA. Anger intensification with combat-related PTSD and depression comorbidity. Psychol Trauma. 2016; 8(1):9-16.

6. Adler AB, Britt TW, Castro CA, McGurk D, Bliese PD. Effect of transition home from combat on risk-taking and health-related behaviors. J Trauma Stress. 2011;24(4):381-9.

7. Novaco RW, Swanson RD, Gonzalez Ol, Gahm GA, Reger MD. Anger and postcombat mental health: validation of a brief anger measure with U.S. soldiers postdeployed from Iraq and Afghanistan. Psychol Assess. 2012;24(3): $661-75$

8. Macmanus D, Dean K, Jones M, Rona RJ, Greenberg N, Hull L, et al. Violent offending by UK military personnel deployed to Iraq and Afghanistan: a data linkage cohort study. Lancet. 2013;381(9870):907-17.

9. Start AR, Allard Y, Adler A, Toblin R. Predicting suicide ideation in the military: the independent role of aggression. Suicide Life Threat Behav. 2019:49(2):444-54.

10. Martin LA, Neighbors HW, Griffith DM. The experience of symptoms of depression in men vs women: analysis of the National Comorbidity Survey Replication. JAMA Psychiatry. 2013;70(10):1100-6.

11. Hoerster KD, Lehavot K, Simpson T, McFall M, Reiber G, Nelson KM. Health and health behavior differences: U.S. military, veteran, and civilian men. Am J Prev Med. 2012:43(5):483-9.

12. Orth $U$, Wieland E. Anger, hostility, and posttraumatic stress disorder in trauma-exposed adults: a meta-analysis. J Consult Clin Psychol. 2006;74(4): 698-706.

13. Cabrera OA, Adler AB, Bliese PD. Growth mixture modeling of post-combat aggression: application to soldiers deployed to Iraq. Psychiatry Res. 2016; 246:539-44. 
14. Rona RJ, Jones M, Hull L, MacManus D, Fear NT, Wessely S. Anger in the UK armed forces: strong association with mental health, childhood antisocial behavior, and combat role. J Nerv Ment Dis. 2015;203(1):15-22.

15. Wilk JE, Quartana PJ, Clarke-Walper K, Kok BC, Riviere LA. Aggression in US soldiers post-deployment: associations with combat exposure and PTSD and the moderating role of trait anger. Aggress Behav. 2015;41(6):556-65.

16. Lloyd D, Nixon RD, Varker T, Elliott P, Perry D, Bryant RA, et al. Comorbidity in the prediction of cognitive processing therapy treatment outcomes for combat-related posttraumatic stress disorder. J Anxiety Disord. 2014;28(2): 237-40.

17. Forbes $D$, Bennett $N$, Biddle D, Crompton D, McHugh T, Elliott P, et al. Clinical presentations and treatment outcomes of peacekeeper veterans with PTSD: preliminary findings. Am J Psychiatry. 2005;162(11):2188-90.

18. Wilk JE, Bliese PD, Thomas JL, Wood MD, McGurk D, Castro CA, et al. Unethical battlefield conduct reported by soldiers serving in the Iraq war. J Nerv Ment Dis. 2013;201(4):259-65.

19. Lerner JS, Li Y, Valdesolo P, Kassam KS. Emotion and decision making. Annu Rev Psychol. 2015;66:799-823.

20. Spielberger CD. State-Trait Anger Expression Inventory. Second Edition: Professional Manual. Lutz: Psychological Assessment Resources; 1999.

21. Forbes D, Alkemade N, Hopcraft D, Hawthorne G, O'Halloran P, Elhai JD, et al. Evaluation of the dimensions of anger reactions-5 (DAR-5) scale in combat veterans with posttraumatic stress disorder. J Anxiety Dis. 2014; 28(8):830-5

22. Forbes D, Alkemade N, Mitchell D, Elhai JD, McHugh T, Bates G, et al. Utility of the dimensions of anger Reactions-5 (DAR-5) scale as a brief anger measure. Depress Anxiety. 2014;31(2):166-73.

23. Ceschi G, Selosse G, Nixon RDV, Metcalf O, Forbes D. Posttraumatic anger: a confirmatory factor analysis of the dimensions of anger reactions Scale-5 (DAR-5) - French adaptation. Eur J Psychotraumatol. 2020;11(1):1731127.

24. Ryan MA, Smith TC, Smith B, Amoroso P, Boyko EJ, Gray GC, et al. Millennium cohort: enrollment begins a 21-year contribution to understanding the impact of military service. J Clin Epidemiol. 2007;60(2): 181-91.

25. Smith TC. The US Department of Defense Millennium Cohort Study: career span and beyond longitudinal follow-up. J Occup Environ Med. 2009;51(10): 1193-201.

26. Crum-Cianflone NF, Fairbank JA, Marmar CR, Schlenger W. The millennium cohort family study: a prospective evaluation of the health and well-being of military service members and their families. Int J Methods Psychiatr Res. 2014;23(3):320-30

27. Littman AJ, Boyko EJ, Jacobson IG, Horton J, Gackstetter GD, Smith B, et al. Assessing nonresponse bias at follow-up in a large prospective cohort of relatively young and mobile military service members. BMC Med Res Methodol. 2010;10:99.

28. Wells TS, Jacobson IG, Smith TC, Spooner CN, Smith B, Reed RJ, et al. Prior health care utilization as a potential determinant of enrollment in a 21-year prospective study, the millennium cohort study. Eur J Epidemiol. 2008;23(2): 79-87.

29. Smith TC, Zamorski M, Smith B, Riddle JR, Leardmann CA, Wells TS, et al. The physical and mental health of a large military cohort: baseline functional health status of the millennium cohort. BMC Public Health. 2007; 7:340.

30. Crum-Cianflone NF, Powell TM, LeardMann CA, Russell DW, Boyko EJ. Mental health and comorbidities in U.S. military members. Mil Med. 2016; 181(6):537-45

31. Asmundson GJ, LeBouthillier DM, Parkerson HA, Horswill SC. Traumaexposed community-dwelling women and men respond similarly to the DAR-5 anger scale: factor structure invariance and differential item functioning. J Trauma Stress. 2016;29(3):214-20.

32. Hoffman J, Liddell B, Bryant RA, Nickerson A. The relationship between moral injury appraisals, trauma exposure, and mental health in refugees. Depress Anxiety. 2018;35(11):1030-9.

33. Murphy D, Ashwick R, Palmer $E$, Busuttil W. Describing the profile of a population of UK veterans seeking support for mental health difficulties. J Ment Health. 2019;28(6):654-61.

34. Armenta RF, Rush T, LeardMann CA, Millegan J, Cooper A, Hoge CW, et al. Factors associated with persistent posttraumatic stress disorder among U.S military service members and veterans. BMC Psychiatry. 2018;18(1).

35. Finkelhor $\mathrm{D}$. The juvenile victimization questionnaire: reliability, validity, and national norms. Child Abuse Negl. 2005;29(4):383-412.
36. Blanchard EB, Jones-Alexander J, Buckley TC, Forneris CA. Psychometric properties of the PTSD checklist (PCL). Behav Res Ther. 1996;34(8):669-73.

37. Association AP. Diagnostic and statistical manual of mental disorders 4th ed. DSM-IV. 4th ed. Washington, DC: American Psychiatric Association; 1994.

38. Spitzer RL, Williams JB, Kroenke K, Linzer M, de Gruy FV 3rd, Hahn SR, et al. Utility of a new procedure for diagnosing mental disorders in primary care. The PRIME-MD 1000 study. JAMA. 1994;272(22):1749-56.

39. Spitzer RL, KK, JBW. Validation and utility of a self-report version of PRIMEMD: the PHQ primary care study. Primary care evaluation of mental disorders. Patient health questionnaire. JAMA. 1999;282(18):1737-44.

40. Cann A, Calhoun LG, Tedeschi RG, Taku K, Vishnevsky T, Triplett KN, et al. A short form of the posttraumatic growth inventory. Anxiety Stress Coping. 2010;23(2):127-37.

41. Kaur N, Porter B, LeardMann CA, et al. Evaluation of a modified version of the Posttraumatic Growth Inventory-Short Form. BMC Med Res Methodol. 2017;17(1):69.

42. Pearlin LI, Schooler C. The structure of coping. J Health Soc Behav. 1978; 19(March):2-21.

43. Lu K. Number of imputations needed to stabilize estimated treatment difference in longitudinal data analysis. Stat Methods Med Res. 2017;26(2): 674-90.

44. Okuda M, Picazo J, Olfson M, Hasin DS, Liu SM, Bernardi S, et al. Prevalence and correlates of anger in the community: results from a national survey. CNS Spectr. 2015;20(2):130-9.

45. Boylan JM, Ryff CD. Varieties of anger and the inverse link between education and inflammation: toward an integrative framework. Psychosom Med. 2013;75(6):566-74.

46. Mikula $\mathrm{G}$, Scherer KR, \& Athenstaedt U. The role of injustice in the elicitation of differential emotional reactions. Personal Soc Psychol Bull. 1998;24(7): 769-83.

47. Cash R, Varker T, McHugh T, Metcalf O, Howard A, Lloyd D, et al. Effectiveness of an anger intervention for military members with PTSD: a clinical case series. Mil Med. 2018;183(9-10):e286-90.

48. Forbes D, Van Hooff M, Lawrence-Wood E, Sadler N, Hodson S, Benassi H, et al. Mental health and wellbeing transition study - pathways to care. Canberra: Department of Veterans' Affairs; 2018. Available from: https://core. ac.uk/download/pdf/158373258.pdf

49. Morland LA, Greene CJ, Rosen CS, Foy D, Reilly P, Shore J, et al. Telemedicine for anger management therapy in a rural population of combat veterans with posttraumatic stress disorder: a randomized noninferiority trial. J Clin Psychiatry. 2010;71(7):855-63.

50. Steenkamp MM, Litz BT, Hoge CW, Marmar CR. Psychotherapy for militaryrelated PTSD: a review of randomized clinical trials. JAMA. 2015;314(5):489500.

51. Lopez AA, Adler AB, Cabrera OA, Thomas JL. Validation of the WRAIR leadership scale. Mil Behav Health. 2018;7(2):125-34.

52. Adler AB, Bliese PD, Pickering MA, Hammermeister J, Williams J, Harada C, et al. Mental skills training with basic combat training soldiers: a grouprandomized trial. J Appl Psychol. 2015;100(6):1752-64.

53. Tedeschi RG, McNally RJ. Can we facilitate posttraumatic growth in combat veterans? Am Psychol. 2011;66(1):19-24.

54. Smith TC, Smith B, Jacobson IG, Corbeil TE, Ryan MA, Millennium cohort study T. Reliability of standard health assessment instruments in a large, population-based cohort study. Ann Epidemiol. 2007;17(7):525-32.

\section{Publisher's Note}

Springer Nature remains neutral with regard to jurisdictional claims in published maps and institutional affiliations. 